\title{
August 2017 Pulmonary Case of the Month
}

Lewis J. Wesselius, MD

\author{
Department of Pulmonary Medicine \\ Mayo Clinic Arizona \\ Scottsdale, AZ USA
}

\section{History of Present IIIness}

The patient is a 60-year-old woman with dyspnea on exertion when she had a pulmonary embolism following knee surgery 3 years earlier. She smoked 1 pack per day for the past 40 years. She was seen at another hospital and had pulmonary function testing which showed only a DLco which was $66 \%$ of predicted. Serologic studies were negative for a rheumatologic disorder. A CT scan was also performed (Figure 1).

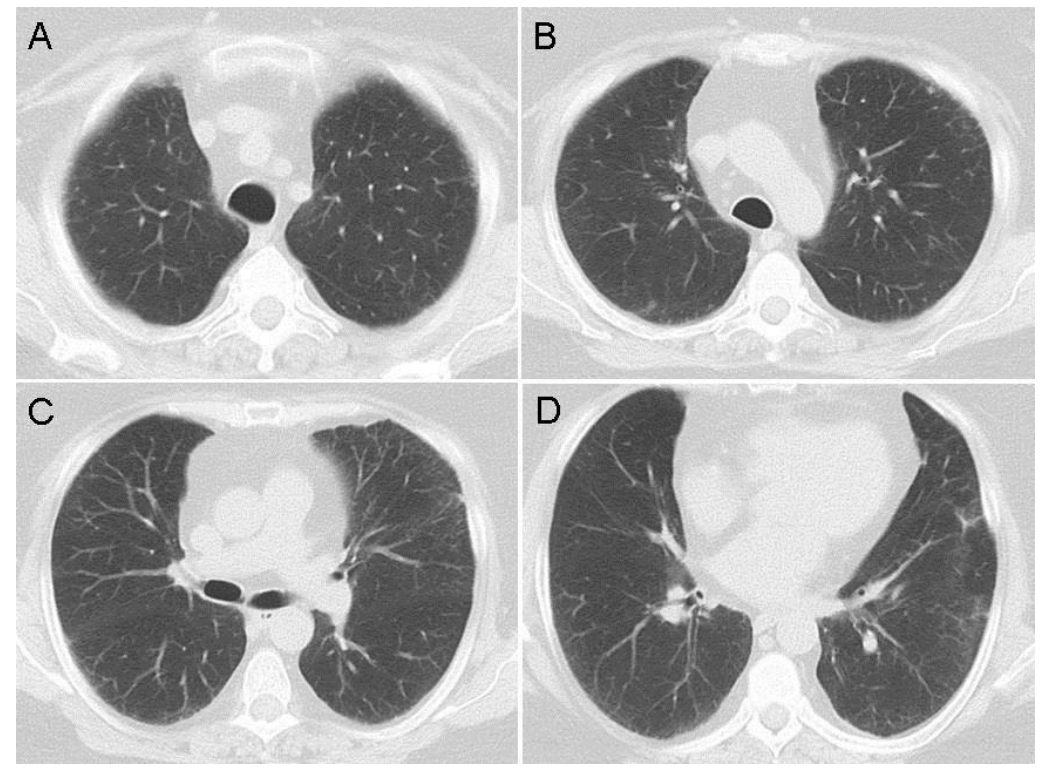

Figure 1. Representative images from thoracic CT scan in lung windows.

The CT scan was interpreted as showing a few small nodules and possible very early interstitial lung disease.

Which of the following are true?

1. A pulmonary embolism can reduce the DLco

2. Her CT scan is characteristic of Langerhans cell histiocytosis

3. Smoking can reduce the DLco

4. 1 and 3

5. All of the above 


\section{Correct!}

\section{1 and 3}

Pulmonary embolism and smoking can both mildly reduce the $\operatorname{DLco}(1,2)$. Pulmonary Langerhans cell histiocytosis has a variable appearance depending on the stage of disease, ranging from small peribronchiolar nodular opacities to multiple irregularly-shaped cysts (3). The earliest change is a diffuse bilateral reticulonodular pattern with a predilection for the mid and upper zones. Later, cyst formation may be seen or may mimic a honeycomb appearance due to a summation of air-filled cysts. Although the CT scan might be compatible with very early Langerhans cell histiocytosis, it is not characteristic.

Her symptoms of mild dyspnea on exertion persist and she is seen 5 years later. Physical examination is unremarkable and a serologic panel for rheumatologic disease is again normal. Her CT scan and pulmonary function testing are repeated (Figures 2 and 3 ).

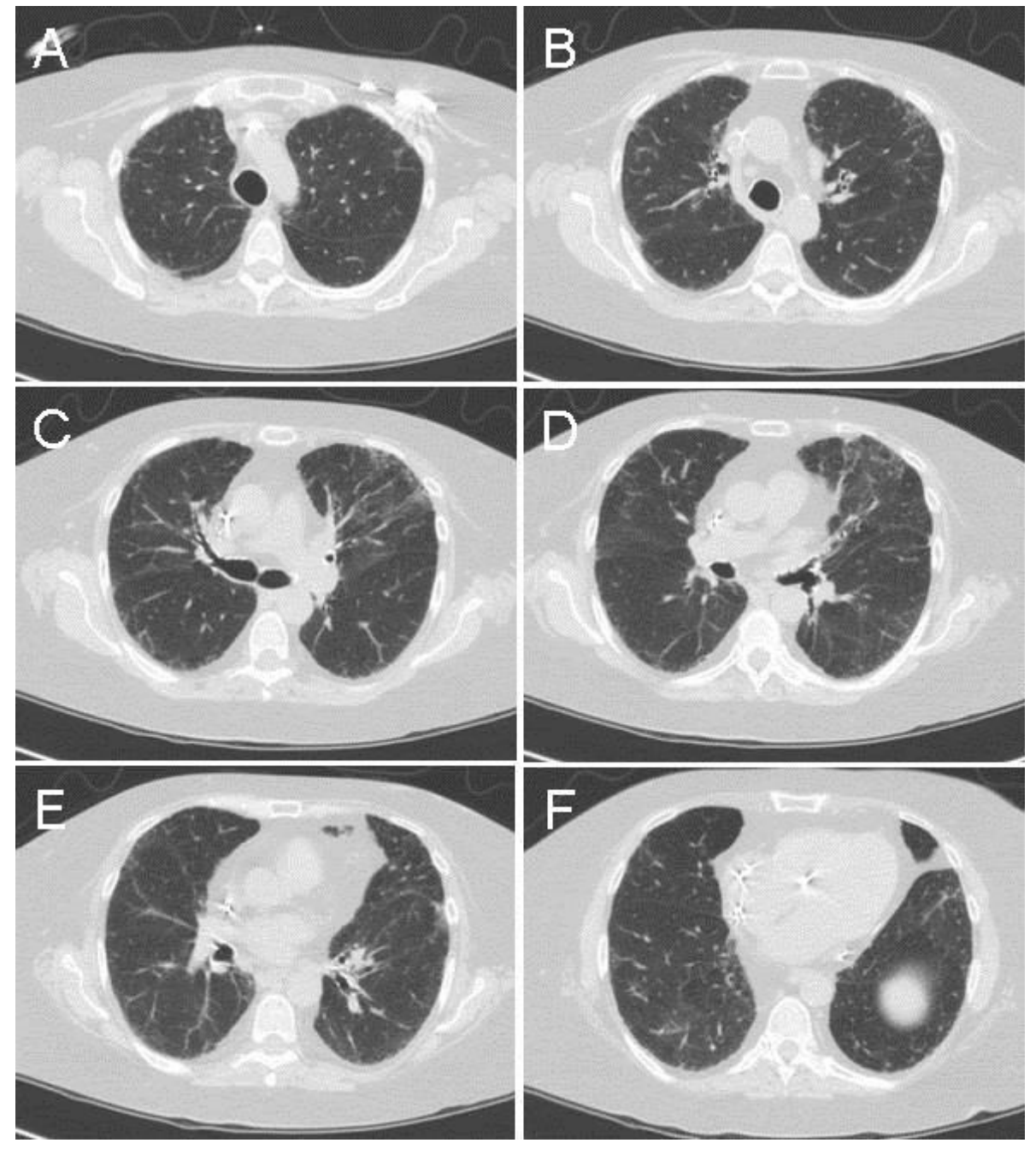

Figure 2. Representative images from the thoracic CT scan in lung windows. 


\section{-- SPIROMETRY --}

$\begin{array}{lrr}\text { FVC (L) } & * 1.72 & * 67 \\ \text { FEV1 (L) } & * 1.51 & * 79 \\ \text { FEV1/FVC (\%) } & 88 & 115 \\ \text { FEF 25-75\% (L/sec) } & 2.07 & 126 \\ \text { FEF Max (L/sec) } & 6.00 & 119 \\ \text { MVV (L/min) } & 73 & 88 \\ & & \\ \text { - DIFFUSION -- } & & \\ \text { DLCOunc }(\mathrm{ml} / \mathrm{min} / \mathrm{mmHg}) & * 12.87 & * 67\end{array}$

Figure 3. Repeat pulmonary function testing.

What should be done at this time?

1. Bronchoscopic cryobiopsy

2. Continue to follow the patient

3. Ventilation-perfusion scan

4. 1 and 3

5. All of the above 


\section{Correct! \\ 2. Continue to follow the patient}

Her symptoms are mild and there has been little apparent change compared to 5 years earlier. Therefore, a decision was made to follow the patient. Ventilationperfusion scanning might show multiple pulmonary emboli but this seems unlikely. Cryobiopsy might show pathology suggesting an interstitial lung disease but seems overly aggressive at this point.

She is again followed and returns 3 years later with her symptoms slightly worsened.

What should be done at this time?

1. Repeat pulmonary function testing

2. Consider video-assisted thorascopic biopsy

3. High resolution CT scan with expiratory views

4. 1 and 3

5. All of the above 


\section{Correct!}

\section{1 and 3}

Again, at this juncture biopsy seems overly aggressive based on her symptoms unless her pulmonary function testing or repeat CT scan show some changes. Pulmonary function testing was unchanged compared to three years earlier. A high resolution scan with inspiratory and expiratory views was performed (Figure 4).

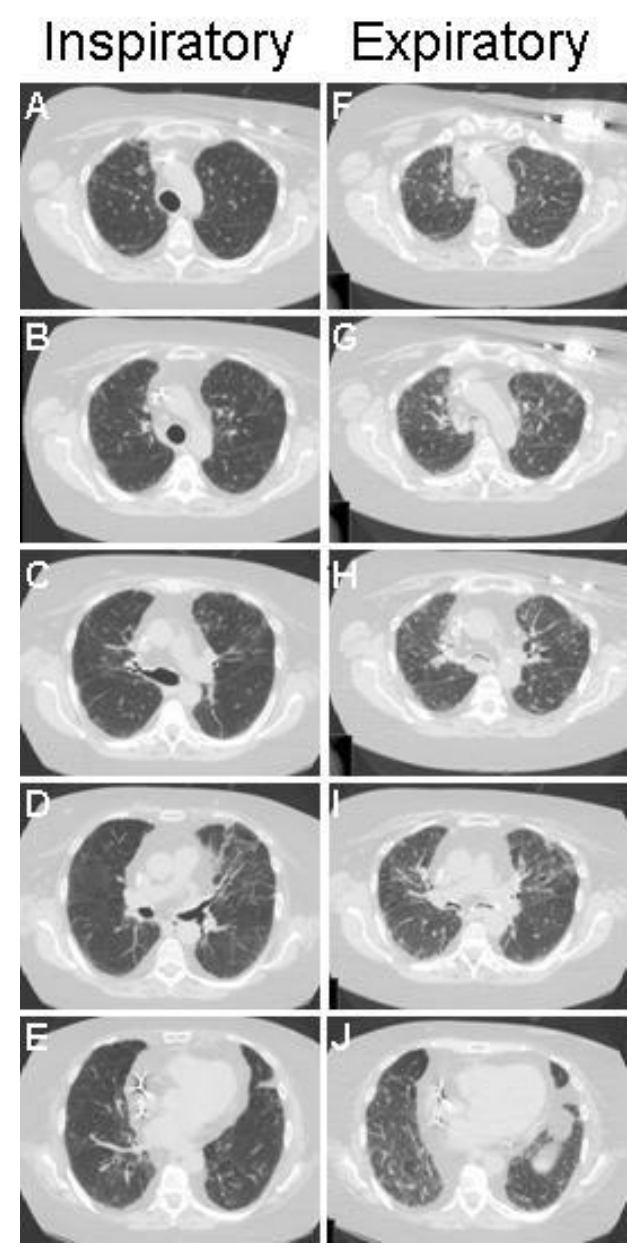

Figure 4. Representative inspiratory images (A-E) and expiratory images (F-J) in lung windows from high-resolution thoracic CT scan.

What is the patient's diagnosis?

1. Chronic obstructive pulmonary disease with air trapping

2. Langerhans cell histiocytosis

3. Nonspecific interstitial pneumonia (NSIP)

4. Tracheobronchomalacia

5. Usual interstitial pneumonia (UIP) 


\section{Correct! \\ 4. Tracheobronchomalacia}

Note the near or total collapse of the trachea and major bronchi during at end expiration. Severe, diffuse tracheobronchomalacia (TBM) is an underrecognized cause of dyspnea, recurrent respiratory infections, cough, secretion retention, and even respiratory insufficiency (3). The posterior wall of the trachea and airways normally collapse during expiration (Figure 5A, 5B). However, when this is excessive $(5 \mathrm{C}, 5 \mathrm{E})$ or when the anterior wall collapses $(5 \mathrm{D}, 5 \mathrm{E})$ it can lead to symptoms.

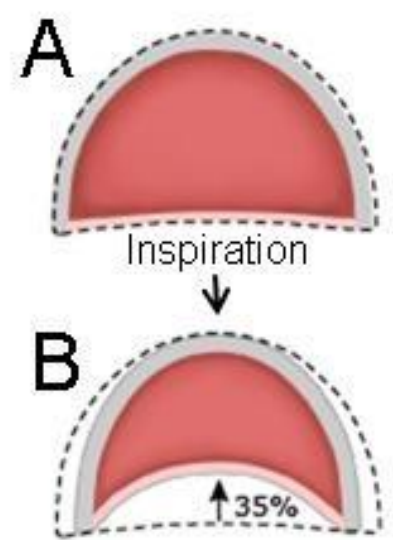

Expiration

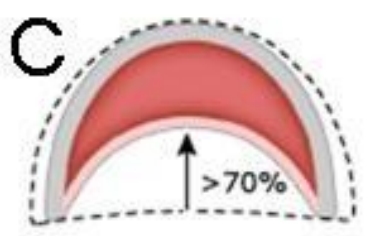

EDAC

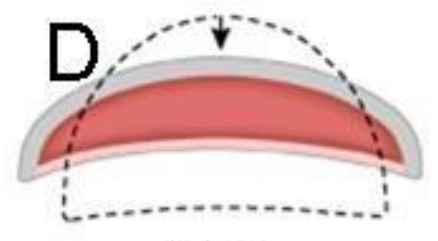

TBM

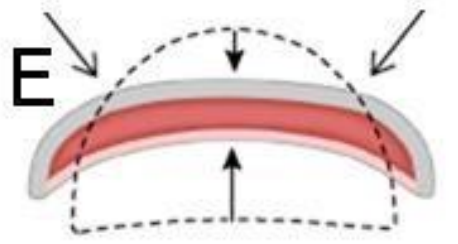

EDAC and TBM

Figure 5. Schematic of normal airway collapse, excessive dynamic airway collapse (EDAC) and tracheomalacia (TBM). Panel A: normal trachea at end inspiration. Panel B: normal trachea at end expiration. Panel C: abnormal trachea showing EDAC at end expiration with the tracheal cartilage supporting the anterior trachea but excessive collapse of the posterior trachea. Panel D: TBM at end expiration showing collapse the anterior trachea and to a lesser extent the posterior trachea. Panel E: EDAC and TBM and end expiration showing collapse of both the anterior and posterior trachea forming a crescent.

The prevailing definition of TBM as a $50 \%$ reduction in cross-sectional area is nonspecific, with a high proportion of healthy volunteers meeting this threshold (4). The clinically significant threshold is complete or near-complete collapse of the airway. TBM may result from relapsing polychondritis or occur in association with airway diseases such as asthma, chronic obstructive pulmonary disease, or cystic fibrosis. However, most of these patients have an acquired form of TBM in which the etiology in unknown. Diagnosis of TBM is made by airway computed tomography scan or flexible bronchoscopy with forced expiration. The prevailing definition of TBM as a 50\% reduction in cross-sectional area is nonspecific, with a high proportion of healthy volunteers meeting this threshold. The clinically significant threshold is complete or near-complete collapse of the airway. 
Airway stenting may treat TBM, although complications resulting from indwelling prostheses often limit the durability of stents (4). Surgical central airway stabilization by posterior mesh splinting (tracheobronchoplasty) effectively corrects malacic airways and has been shown to lead to significant improvement in symptoms. A short-term tracheal stent trial facilitates surgical selection.

After hearing the pros and cons of therapy, our patient chose to be followed rather than undergo tracheobronchoplasty.

\section{References}

1. Elliott CG. Pulmonary physiology during pulmonary embolism. Chest. 1992;101(4):163S-171S. [CrossRef] [PubMed]

2. Sansores RH, Pare PD, Abboud RT. Acute effect of cigarette smoking on the carbon monoxide diffusing capacity of the lung. Am Rev Respir Dis. 1992 Oct;146(4):951-8. [CrossRef] [PubMed]

3. Brauner MW, Grenier P, Tijani K, Battesti JP, Valeyre D.Pulmonary Langerhans cell histiocytosis: evolution of lesions on CT scans. Radiology. 1997 Aug;204(2):497-502. [CrossRef] [PubMed]

4. Buitrago DH, Wilson JL, Parikh M, Majid A, Gangadharan SP. Current concepts in severe adult tracheobronchomalacia: evaluation and treatment. J Thorac Dis. 2017 Jan;9(1):E57-E66. [CrossRef] [PubMed] 